PHYSICAL REVIEW A 72, 069909(E) (2005)

\title{
Publisher's Note: Generalized universal cloning and purification in quantum information by multistep state symmetrization [Phys. Rev. A 72, 060304 (2005)]
}

L. Masullo, M. Ricci, and F. De Martini

(Received 13 December 2005; published 21 December 2005)

DOI: 10.1103/PhysRevA.72.069909

PACS number(s): 03.67.-a, 42.50.Dv, 99.10.Fg

This paper was published online on 9 December 2005 with an incorrect copyright year in the headers and footers. The year should read "2005." The copyright has been corrected as of 13 December 2005. The copyright is correct in the printed version of the journal. 